\title{
Boundary Set Based Existence Recognition and Construction of Hypertree Agent Organization
}

\author{
Yang Xiang and Kamala Srinivasan \\ School of Computer Science, University of Guelph, Canada
}

\begin{abstract}
Some of the essential tasks of a multiagent system (MAS) include distributed probabilistic reasoning, constraint reasoning, and decision making. Junction tree (JT) based agent organizations have been adopted by some MAS frameworks for their advantages of efficient communication and sound inference. In addition, JT organizations have the potential capacity to support a high degree of agent privacy. This potential, however, has not been fully realized. We present two necessary and sufficient conditions on the existence of JT organization given a MAS. Following these conditions, we propose a new algorithm suite, based on elimination in the so called boundary set of a MAS, that recognizes JT organization existence and constructs one if exists, while guaranteeing agent privacy on private variables, shared variables and agent identities.
\end{abstract}

\section{Introduction}

Some of the essential tasks of a multiagent system (MAS) include distributed probabilistic reasoning, constraint reasoning, and decision making (decision theoretic). Existing frameworks include AEBN [8] and MSBN [10] for probabilistic reasoning, ABT [4], ADOPT [5], DPOP [7], Action-GDL [9], DCTE [1], and MSCN [12] for constraint reasoning, and RMM [2], MAID [3], and CDN [11] for decision making. Frameworks such as AEBN and MAID do not assume specific agent organization. ABT assumes a total order among agents. ADOPT and DPOPS use a pseudo-tree organization. MSBN, MSCN, CDN, Action-GDL, and DCTE all use a junction tree (JT) organization (known as hypertree in the first three frameworks), which is the focus of this work.

In JT-based frameworks, the application environment is represented by a set of variables, referred to as the env. The env is decomposed into a set of overlapping subenvs, each being a subset of env. The subenvs are one-to-one mapped to agents of the MAS. Subenvs (and hence agents) are organized into a JT. A JT is a tree where each node is associated with a set of variables called a cluster. The tree is structured such that, the intersection of any two clusters is contained in every cluster on the path between the two (running intersection). In a JT agent organization, each cluster corresponds to a subenv and its agent. The organization prescribes communication pathways: an agent can send a message to another, iff they are adjacent in the JT.

JT-based agent organizations support several desirable properties. First, they allow efficient communication. For agents to cooperate by utilizing information available locally at individual agents, it suffices to pass two messages along each link of the JT. Hence, the time complexity of communication is linear in the number of agents. Second,

O. Zaïane and S. Zilles (Eds.): Canadian AI 2013, LNAI 7884, pp. 187-198 2013.

(c) Springer-Verlag Berlin Heidelberg 2013 
they support sound inference. Global consistency is guaranteed by local consistency, e.g., probabilistic reasoning in MSBN [10] is exact, constraint reasoning in MSCN [12] is complete, and decision making in CDN [11] is globally optimal.

Third, the above two properties are enabled while message contents involve only shared variables (those in the intersection of subenvs). Hence, JT organizations have the potential capacity to support a high degree of agent privacy. However, such potential has not been fully realized in existing JT-based MAS frameworks.

In Action-GDL [9], the JT is built through a centralized mapping operation from a pseudo-tree where each node is an env variable. The centralized mapping discloses identities of all variables to the mapping agent. The JT in DCTE [1] is constructed according to [6], where clusters (one per agent) of env variables are initially organized into a tree that is generally not a JT. Variable identities are passed along the tree links to transform the tree into a JT, and hence are disclosed beyond the agent initially associated with them.

In MSBN [10], MSCN [12], and CDN [11] frameworks, the JT organization is constructed by a coordinator agent with knowledge of variables shared by agents. Since coordinator knows nothing about private variables (those that are contained in a single subenv), their privacy is ensured. However, shared variables and agent identities are disclosed to the coordinator.

The contribution of this work is a new algorithm suite for JT organization based on the so-called boundary-set (see below) of the MAS. Given the boundary-set of a MAS, the algorithm first determines distributively whether a JT organization exists. If it does, then a JT will be constructed. The entire process preserves agent privacy on private variables, shared variables, and agent identities. To the best of our knowledge, no known JT-based MAS frameworks provide the same degree of agent privacy, except an alternative approach for distributed JT construction that we report in a related work [13].

The next section defines the problem that we tackle. The subsequent section presents a necessary and sufficient condition on the existence of JT organization. This insight allows a classification of MAS subenv decompositions relative to JT organization existence. The next section gives another necessary and sufficient condition on JT organization existence, which leads to an agent privacy preserving algorithm suite for JT organization existence recognition and construction, illustrated and then specified in the following two sections.

\section{Problem Definition}

Consider a MAS populated by a set $\mathscr{A}=\left\{A_{0}, \ldots, A_{\eta-1}\right\}$ of $\eta>1$ agents. Let $V$ be the set of env variables, and be decomposed into a collection of subenvs, $\Omega=\left\{V_{0}, \ldots, V_{\eta-1}\right\}$, such that $\cup_{i=0}^{\eta-1} V_{i}=V$. Each $V_{i}$ is associated with a unique agent $A_{i}$, and vice versa. We refer to the set of shared variables, $I_{i j}=V_{i} \cap V_{j} \neq \emptyset$, between $A_{i}$ and $A_{j}$ as their border. For each agent $A_{i}$, we denote the set $W_{i}=\cup_{j \neq i} I_{i j}$ as its boundary, and we refer to $W=\left\{W_{0}, \ldots, W_{\eta-1}\right\}$ as the boundary set of the MAS. Fig.11(a) shows a subenv decomposition of a trivial env, with agent boundaries shown in (b). $A_{i}$ knows $A_{j}$ and can communicate with $A_{j}$, iff they have a border. The message between them can only involve variables included in their border. 


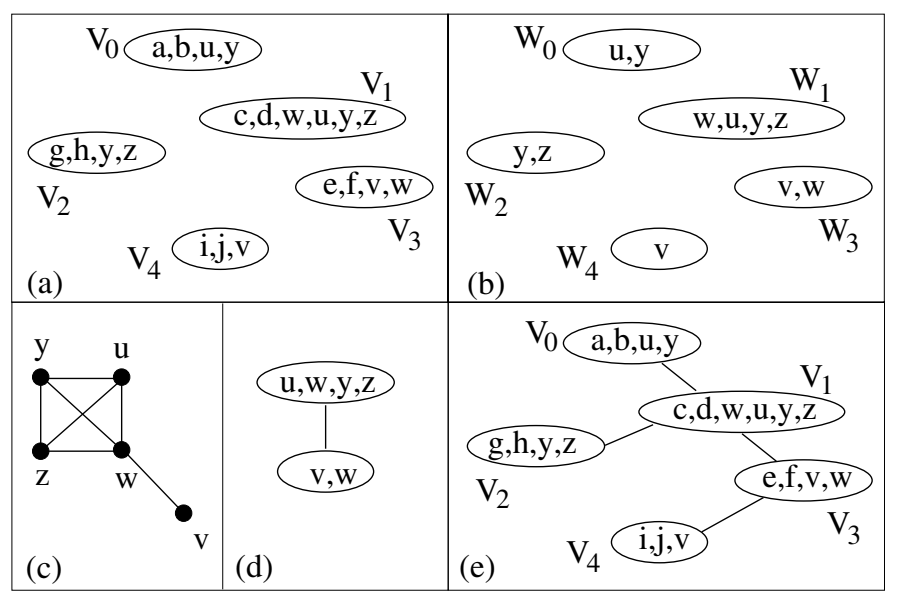

Fig. 1. (a) Subenv decomposition. (b) Agent boundaries. (c) Boundary graph. (d) JT from (c). (e) JT organization for (a).

The general task of JT agent organization is to construct a JT with subenvs as clusters such that running intersection holds.

A variable $x \in V$ is private if $x$ is contained in a unique subenv $V_{i}$. Agent privacy on private variables is preserved, if no information on any private variable is disclosed to other agents, including its existence, identity, domain (of possible values), associated conditional or marginal probability distribution (in the case of probabilistic reasoning), or constraint (in the cases of constraint reasoning and decision making), and observed or assigned value.

A variable $x \in V$ is shared by $A_{i}$ and $A_{j}$, if $x \in I_{i j}$. Agent privacy on shared variables is preserved, if no information on any shared variable is disclosed beyond agents who share it.

An agent is known to another agent, iff they share a border. Privacy on agent identities is preserved, if for every agent, its identity is not disclosed to any non-bordering agent.

The boundary set of a MAS can play an important role in privacy preserving construction of JT organizations, as established by a proposition from [13].

Proposition 1. Let $V$ be env of a MAS, $\Omega$ be its subenv decomposition, $W$ be the boundary set, and $T$ be a JT with boundaries in $W$ as clusters. Let $T^{\prime}$ be a cluster tree with subenvs of $\Omega$ as clusters, such that it is isomorphic to $T$ with each subenv cluster mapped to the corresponding boundary cluster in $T$. Then $T^{\prime}$ is a JT.

Based on Prop.11 we take the approach to construct a JT organization from the boundary set $W$, rather than from the subenv decomposition $\Omega$. This approach guarantees agent privacy on private variables, because these variables are excluded from input of the task.

Given a boundary set, a JT made of its boundary clusters may or may not exist. Hence, the problem we tackle in this work is stated as follows. Given the boundary set of a MAS, determine whether a JT agent organization exists and, if so, construct a JT, 
such that agent privacy on private variables, shared variables, and agent identities are preserved in the process.

\section{Boundary Graph Based Condition on Hypertree Existence}

Before the existence of JT organization can be determined algorithmically, we analyze conditions of its existence, through an alternative representation of the boundary set. Let $W$ be the boundary set of a MAS. An undirected graph $B G$ is the boundary graph of the MAS, if its set of nodes is $N=\cup_{i=0}^{\eta-1} W_{i}$, and its links are connected so that each $W_{i}$ is complete (elements are pairwise connected).

Prop.2 identifies a condition under which a JT can be constructed from a boundary graph such that each JT cluster is a boundary. A set of nodes in a graph is a clique, if they are maximally pairwise connected. Two clusters are comparable, if one is a subset of the other.

Proposition 2. Let $W$ be the boundary set of a MAS, and BG be its boundary graph, such that

1. BG is chordal, and

2. for each clique $C$ of $B G$, there exists $W_{i} \in W$ with $C \subseteq W_{i}$.

Let $T$ be a JT whose clusters are cliques of BG, and no two clusters in $T$ are comparable. Then for every cluster $C$ of $T$, there exists a boundary $W_{i}=C$.

Proof: From subcondition 1, the JT $T$ exists. We prove by contradiction. Suppose there exists a cluster $C$ in $T$ such that $C \neq W_{i}$ for every $W_{i} \in W$.

From subcondition 2, there exists $W_{i}$ such that $C \subseteq W_{i}$. Since $C \neq W_{i}$, it follows that $C \subset W_{i}$. Because $B G$ is a boundary graph, $W_{i}$ is complete in $B G$. Therefore, there exists a cluster $C_{i}$ in $T$ such that $W_{i} \subseteq C_{i}$. From $C \subset W_{i}$ and $W_{i} \subseteq C_{i}$, we have $C \subset C_{i}$. That is, $T$ contains two comparable clusters: a contradiction. Hence, every cluster in $T$ is a boundary.

Example 1. Fig. П (a) shows an env decomposition. The set of boundaries is shown in (b). The BG is shown in (c), and it satisfies the two conditions. The JT from the BG is shown in $(d)$, where the two clusters are boundaries $W_{1}$ and $W_{3}$.

Utilizing Prop. 2. Theorem 1 establishes a necessary and sufficient condition for the existence of JT organization.

Theorem 1. Let $W$ be the boundary set of a MAS and BG be its boundary graph. A JT agent organization exists, iff the following hold.

1. $B G$ is chordal, and

2. for each clique $C$ of $B G$, there exists $W_{i} \in W$ such that $C \subseteq W_{i}$. 
Proof: [Necessity] Suppose a JT $H$ exists, whose clusters are subenvs. For each cluster in $H$, remove its private variables. The resultant cluster tree $T$ is still a JT, and its corresponding undirected graph is $B G$. From $T$ being a JT, it follows that $B G$ is chordal. Hence, subcondition 1 holds. The clusters of $T$ are one-to-one mapped to boundaries of agents, from which subcondition 2 follows.

[Sufficiency] Suppose both subconditions hold. We prove by construction.

Since $B G$ is chordal, a JT $T$ exists whose clusters are cliques of $B G$. Without losing generality, assume that clusters of $T$ are not comparable. By Prop. 2, every cluster in $T$ is a boundary. Hence, for every cluster $C$ such that $C=W_{i}$ for some $i$, we can associate $C$ with an agent $A_{i}$.

If not every agent is associated with a cluster yet, consider a remaining agent $A_{i}$ without being associated with any cluster of $T$ yet. Since $W_{i}$ is complete in $B G$, there exists a cluster $C$ in $T$ such that $W_{i} \subseteq C$. Add to $T$ a new cluster $W_{i}$, make it adjacent to cluster $C$, and associate the new cluster with $A_{i}$. Repeat this for each remaining agent, until each agent is associated with a cluster in $T$.

Next, for each agent, add its private variables to its associated cluster in $T$. The resul$\operatorname{tant} T$ is a JT agent organization with each cluster being a subenv.

Theorem 1 provides the following insight. As far as the existence of JT organization is concerned, MAS subenv decompositions can be classified into three types.

Type 1 Boundary graphs are chordal, and their cliques are boundary contained.

Type 2 Boundary graphs are not chordal.

Type 3 Boundary graphs are chordal, but their cliques are not boundary contained.

Example 2. The boundary graph for the subenv decomposition in Fig. 1 (a) is shown in (c). The subenv decomposition is type 1. The JT of the boundary graph is show in (d). The two clusters are associated with $A_{1}$ and $A_{3}$. For each of the three remaining agents, a cluster can be added to the JT. The JT organization is shown in (e).

Example 3. Fig. 2 (a) shows another subenv decomposition, with agent boundaries in (b) and BG in (c). Since BG is not chordal, the subenv decomposition is type 2. By Theorem 1 it has no JT organization.

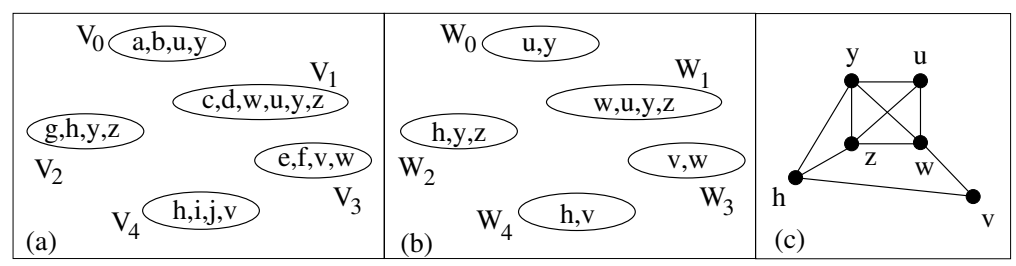

Fig. 2. (a) Subenv decomposition. (b) Agent boundaries. (c) Boundary graph.

Example 4. For agent boundaries in Fig. 3 (a), the BG is shown in (b). It has two cliques. Since one of them, $\{h, v, w\}$, is not contained in any boundary, the subenv decomposition is type 3. By Theorem 1 it has no JT organization. 


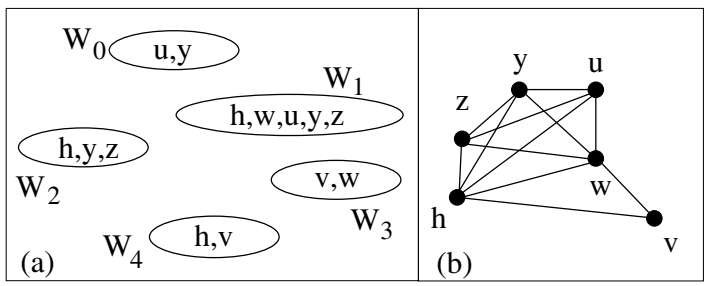

Fig. 3. (a) Agent boundaries. (b) Boundary graph.

\section{Boundary Set Based Condition of Hypertree Existence}

First, we define an operation to eliminate a boundary from a boundary set $W$. When a boundary $W_{i} \in W$ is eliminated from $W$ relative to boundary $W_{j} \in W$, where $W_{i} \cap W_{j} \neq \emptyset$, it yields a reduced boundary set $W^{\prime}=\left(W \backslash\left\{W_{i}, W_{j}\right\}\right) \cup\left\{W_{j}^{\prime}\right\}$, where

$$
W_{j}^{\prime}=\bigcup_{W_{k} \in W, k \neq i, k \neq j}\left(W_{j} \cap W_{k}\right) .
$$

That is, the set $W^{\prime}$ resultant from eliminating $W_{i}$ relative to $W_{j}$ is obtained by deleting $W_{i}$ and $W_{j}$ from $W$, and replacing with $W_{j}^{\prime}$. The $W_{j}^{\prime}$ is obtained by the union of borders of $A_{j}$, except the border with $A_{i}$. In other words, $W_{j}^{\prime}$ is the boundary $W_{j}$ without variables that $A_{j}$ uniquely shares with $A_{i}$. Consider the boundary set for Fig. 10 (b), $W=\left\{W_{0}, \ldots, W_{4}\right\}$. After $W_{0}$ is eliminated relative to $W_{1}$, the reduced boundary set is $W^{\prime}=\left\{W_{1}^{\prime}, W_{2}, W_{3}, W_{4}\right\}$, where $W_{1}^{\prime}=\{w, y, z\}$.

Without confusion, we refer to each element of $W^{\prime}$ as a boundary, whether or not it is identical to an element of the original boundary set. The elimination operation is well defined on the reduced boundary set, and hence can be performed iteratively.

Example 5. For the boundary set of Fig. 1 elimination can be performed iteratively as follows.

$$
\begin{gathered}
W=\left\{W_{0}=\{u, y\}, W_{1}=\{w, u, y, z\}, W_{2}=\{y, z\}, W_{3}=\{v, w\}, W_{4}=\{v\}\right\} ; \\
\text { Eliminate }\{u, y\} \text { wrt }\{w, u, y, z\}: W^{\prime}=\{\{w, y, z\},\{y, z\},\{v, w\},\{v\}\} ; \\
\text { Eliminate }\{v\} \text { wrt }\{v, w\}: W^{\prime}=\{\{w, y, z\},\{y, z\},\{w\}\} ; \\
\text { Eliminate }\{w\} \text { wrt }\{w, y, z\}: W^{\prime}=\{\{y, z\},\{y, z\}\} ; \\
\text { Eliminate }\{y, z\} \text { wrt }\{y, z\}: W^{\prime}=\{\{y, z\}\} .
\end{gathered}
$$

Note that, each $W_{i}$ eliminated relative to a $W_{j}$ has been so chosen to satisfy $W_{i} \subseteq W_{j}$. The significance of such a choice will be seen below.

Note also that each reduced boundary set $W^{\prime}$ (except the final singleton) is a welldefined boundary set, in the sense that each variable is shared by at least two boundaries in $W^{\prime}$. Take $W^{\prime}=\{\{w, y, z\},\{y, z\},\{w\}\}$ for example, each of $w, y$, and $z$ is shared by two boundaries. 
Next, we establish another necessary and sufficient condition on hypertree existence, based on boundary elimination.

Theorem 2. A MAS with the boundary set $W$ has a JT agent organization, iff $W$ can be eliminated iteratively into a singleton, such that each $W_{i}$ eliminated relative to a $W_{j}$ satisfies $W_{i} \subseteq W_{j}$.

Sketch of proof: For necessity, suppose a JT $H$ exists. Remove private variables in each cluster. The resultant cluster tree $T$ is a JT, whose set of clusters is $W$. A leaf cluster satisfying the condition can be found in $T$, and eliminated iteratively.

For sufficiency, suppose $W$ can be eliminated into a singleton. Denote the sequence of reduced boundary sets as $W^{\eta}, W^{\eta-1}, \ldots, W^{2}, W^{1}$, where $W^{\eta}=W, W^{1}$ is the final singleton, and the superscript indicates the number of boundaries in the set. Boundaries in each $W^{x}$, for $x=2, \ldots, \eta$, can be organized into a JT.

\section{Distributed Recognition of Hypertree Existence}

The condition $W_{i} \subseteq W_{j}$ in Theorem 2 is equivalent to $W_{i}=I_{i j}$. Hence, Theorem 2 suggests a privacy preserving, distributed computation to identify JT organization existence. Agents are self-eliminated one by one as long as possible. An agent $A_{i}$ can be eliminated if its boundary is equal to the border with another remaining agent $A_{j}$. After $A_{i}$ is eliminated relative to $A_{j}, A_{j}$ removes from its boundary the variables that it shares uniquely with $A_{i}$. If all agents are eliminated except one, then a JT organization exists for the MAS. Otherwise, the JT does not exist.

We assume that a token is passed between bordering agents, according to depthfirst-traversal. The first round of traversal starts at an arbitrary agent, who possesses the token $t^{1} k^{1}$. If an agent $A_{i}$ who holds the token has its boundary equal to the border with another agent $A_{j}$, then $A_{i}$ signifies to each bordering agent that it is eliminated, and passes a new token $t o k^{2}$ to $A_{j}$.

$A_{j}$ then starts the second round of traversal among remaining agents, using $t o k^{2}$. If an agent starts a new round of traversal, and finds that it has no uneliminated bordering agent, then it announces existence of a JT organization.

On the other hand, suppose an agent $A_{j}$ starts a new round of traversal, with at least another uneliminated bordering agent. After the token has traversed every uneliminated agent, and comes back to $A_{j}$, if $A_{j}$ still has uneliminated bordering agents, then $A_{j}$ announces non-existence of JT organization.

Example 6. Consider agents in Fig. 4(a) with their boundaries shown in ovals, where each link shows a bordering relation. Note that the subenv decomposition is type 1.

Suppose $A_{0}$ starts first round with tok ${ }^{1}$. It announces its elimination and passes the token to $A_{1}$. In response, $A_{1}$ reduces its boundary, as in (b), and starts second round with tok ${ }^{2}$. It passes tok ${ }^{2}$ to $A_{2}$. $A_{2}$ announces its elimination and passes to ${ }^{3}$ to $A_{1}$. In response, $A_{1}$ reduces its boundary again, as in (c), and starts third round. It announces its elimination and passes tok ${ }^{4}$ to $A_{3}$. In response, $A_{3}$ reduces its boundary, as in $(d)$, and starts fourth round. It announces its elimination and passes tok ${ }^{5}$ to $A_{4}$. Finally, $A_{4}$ announces existence of a JT organization. 


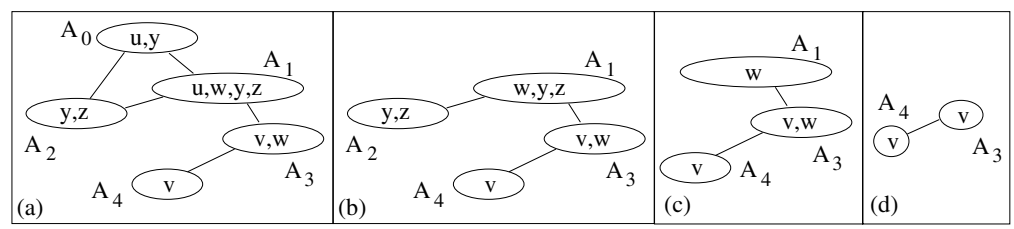

Fig. 4. Distributed recognition of JT organization with type 1 subenv decomposition

Example 7. Consider agents and their boundaries in Fig. 5(a). Note that the subenv decomposition is type 2.

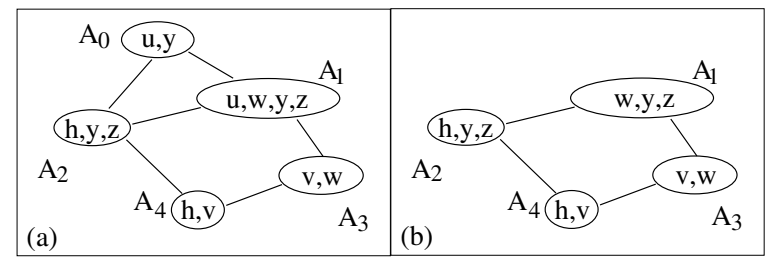

Fig. 5. Recognition of non-existence of JT organization with type 2 subenv decomposition

Suppose $A_{0}$ starts first round with tok ${ }^{1}$, announces its elimination, and passes tok ${ }^{2}$ to $A_{1} . A_{1}$ reduces its boundary, as in (b), starts second round, and passes tok ${ }^{2}$ to $A_{2}$. $A_{2}$ passes tok ${ }^{2}$ to $A_{4}$, who in turn passes tok ${ }^{2}$ to $A_{3}$. $A_{3}$ has no unvisited agent to pass the token to, and returns tok ${ }^{2}$ to $A_{4}$. $A_{4}$ returns tok ${ }^{2}$ to $A_{2}$, who in turn returns to $A_{1}$. Finally, $A_{1}$ announces non-existence of JT organization.

Example 8. Consider agents and their boundaries in Fig. 6(a). Note that the subenv decomposition is type 3.

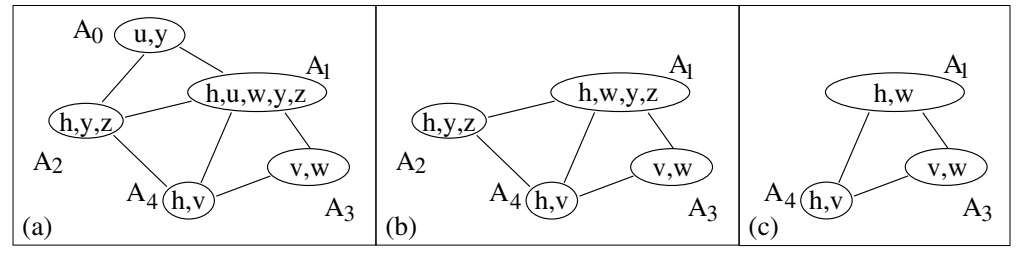

Fig. 6. Recognition of non-existence of JT organization with type 3 subenv decomposition

Suppose $A_{0}$ starts first round with tok ${ }^{1}$, and passes tok ${ }^{2}$ to $A_{1} . A_{1}$ reduces its boundary, as in (b), starts second round, and passes to ${ }^{2}$ to $A_{2} . A_{2}$ announces its elimination and passes tok ${ }^{3}$ to $A_{1}$. $A_{1}$ further reduces its boundary, as in (c), starts third round, 
and passes tok ${ }^{3}$ to $A_{3} . A_{3}$ passes tok ${ }^{3}$ to $A_{4}$. Eventually, tok ${ }^{3}$ is returned to $A_{1}$, who announces non-existence of JT organization.

Note that during the traversal, although the active boundary for a remaining agent may be reduced, the border between any pair of agents never changes.

\section{Algorithm for Hypertree Existence Recognition and Construction}

Next, we specify a distributed algorithm suite, that agents execute to implement the computation described intuitively in the previous section. Each agent's activities are driven by responding to the following messages.

- A StartNewDFT(tok) request that calls the receiver to start a new round of depthfirst-traversal with the given token $t o k$;

- An Eliminated notification sent by an agent who has been self-eliminated;

- A DFT(tok) request that calls the receiver to perform depth-first-traversal with the given token $t o k$;

- A Report message sent by an agent who has been called to perform DFT(tok), signifying either the called agent has been visited in the current round, or it has completed DFT and now backtracks to the caller.

We refer to the receiving agent of a message by $A_{i}$, who will act in response, and refer to the message sender by $A_{c}$. Every agent performs Init to initialize local data. Flag state $\in$ $\{I N, O U T\}$ indicates whether $A_{i}$ has been eliminated. Flag $n b s t a\left(A_{k}\right) \in\{I N, O U T\}$ indicates the same for a bordering agent. Variable curtok keeps a token value after it has visited $A_{i}$, and visited $\left(A_{k}\right)$ indicates whether the token has visited the bordering agent. $Y_{i}$ maintains the active boundary of $A_{i}$.

\section{Procedure 1 (Init)}

1 state $=I N$; parent $=$ null;

2 initialize current token to curtok $=$ null;

3 set active boundary $Y_{i}=W_{i}$;

4 for each bordering agent $A_{k}$,

$5 \quad \operatorname{nbsta}\left(A_{k}\right)=I N$;

$6 \quad \operatorname{visited}\left(A_{k}\right)=$ false;

At the start of each round of traversal, a remaining agent will be be messaged to StartNewDFT. In the first round, an arbitrary leader agent messages itself. Agent $A_{i}$ being messaged does the following.

\section{Procedure 2 (StartNewDFT(tok))}

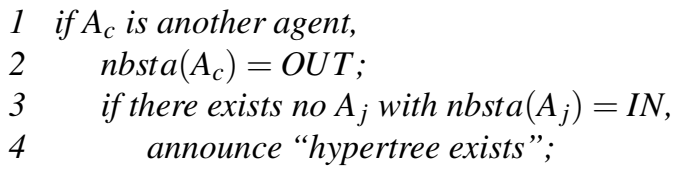




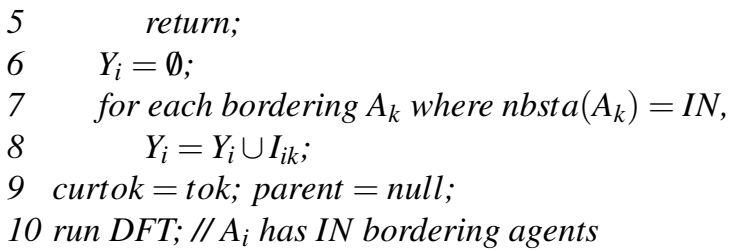

When DFT below is run from StartNewDFT(tok), $A_{i}$ has parent $=n u l l$, and have at least one remaining bordering agent $A_{j}$. If $A_{i}$ can be eliminated relative to $A_{j}$, it will message $A_{j}$ to StartNewDFT. Otherwise, it will send message $D F T$ (tok) to $A_{j}$.

When $D F T$ is run from $D F T$ (tok) (see below), $A_{i}$ has parent pointing to $A_{c}$, and may have no unvisited, remaining bordering agent other than $A_{c}$. If $A_{i}$ cannot be eliminated relative to $A_{c}$, it must Report to $A_{c}$.

\section{Procedure 3 (DFT)}

1 if there exists $A_{j}$ with nbsta $\left(A_{j}\right)=I N$ and $Y_{i}=I_{i j}$, // self-eliminate

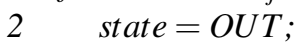

3 for each $A_{k} \neq A_{j}$ where nbsta $\left(A_{k}\right)=I N$, send Eliminated to $A_{k}$;

4 send StartNewDFT(curtok +1$)$ to $A_{j}$;

5 else // no IN agent satisfies $Y_{i}=I_{i j}$

$6 \quad$ for each $A_{k} \neq$ parent where $n b s t a\left(A_{k}\right)=I N$, set visited $\left(A_{k}\right)=$ false;

$7 \quad$ if there exists $A_{k} \neq$ parent where $n b s t a\left(A_{k}\right)=I N$ and visited $\left(A_{k}\right)=$ false,

$8 \quad$ send $A_{k}$ message DFT(curtok);

9 else send Report to parent;

When a remaining agent $A_{i}$ receives from $A_{c}$ the Eliminated message, it responds by setting its $n b s t a\left(A_{c}\right)=O U T$. When a remaining agent $A_{i}$ receives from $A_{c}$ the DFT (tok) message, it performs the following.

\section{Procedure 4 (DFT(tok))}

1 if curtok $=$ tok, // visited

2 send Report to $A_{c}$;

3 else // $A_{i}$ has not seen tok before and has IN bordering agent other than $A_{c}$

$4 \quad$ curtok $=$ tok;

$5 \quad$ parent $=A_{c} ; \operatorname{visited}\left(A_{c}\right)=$ true;

6 run DFT;

After $A_{i}$ has messaged $A_{j}$ with $D F T(t o k)$, it may receive a Report from $A_{j}$. In response, $A_{i}$ performs the following.

\section{Procedure 5 (Respond to Report)}

1 visited $\left(A_{j}\right)=$ true;

2 if there exists $A_{k} \neq$ parent such that $n b s t a\left(A_{k}\right)=I N$ and visited $\left(A_{k}\right)=$ false,

3 select $A_{k}$ to send message DFT(curtok) to it;

4 else // no unvisited bordering agent

$5 \quad$ if parent $=$ null, announce "no hypertree exists"; // DFT starter

$6 \quad$ else send Report to parent; 
The algorithm suite, we refer to as HTBS, terminates when a remaining agent announces "hypertree exists" or otherwise. Its soundness and completeness is established below, which follows from Theorem 2 .

Corollary 1. A MAS with the boundary set W has a JT agent organization, iff HTBS terminates with announcement "hypertree exists". Otherwise, HTBS terminates with announcement "no hypertree exists".

An important product of HTBS is the JT organization emerging upon positive announcement. For every agent $A_{i}$ self-eliminated relative to $A_{j}, A_{i}$ is the sender of StartNewDFT message and $A_{j}$ is the receiver. This relation implies that they are adjacent in the JT organization. Readers are encouraged to verify this by comparing Example 6 with Fig. 1 (e). This result is summarized below, whose proof is omitted due to space limitation.

Theorem 3. If a MAS with the boundary set W has a JT agent organization, then agent adjacency in the JT is defined by StartNewDFT sender-receiver relation during HTBS.

Let $e$ be the number of pairs of bordering agents. In each round of HTBS, at most $O(e)$ messages are passed. HTBS halts in at most $O(\eta)$ rounds. Hence, its time complexity is $O(e \eta)$.

Since HTBS is based on boundary set, agent privacy on private variables is guaranteed. Since HTBS messages contains no information on shared variables, agent privacy on shared variables is guaranteed. Since HTBS messages are passed between bordering agents only, and the message argument is a token only, privacy on agent identity is guaranteed.

\section{Conclusion}

The contributions of this research include the following. We proved two necessary and sufficient conditions for the existence of a JT organization given a MAS subenv decomposition. One of them provides insight and classification of subenv decompositions, and the other suggests a distributed reorganization of JT organization existence. Based on the second condition, we have presented an algorithm suite that recognize JT organization existence and construct it if exists. The algorithm guarantees agent privacy on private variables, shared variables, as well as agent identity. To the best of our knowledge, no existing JT-based MAS frameworks provide the same degree of agent privacy, except a related work based on distributed maximum spanning tree construction which we report in [13].

Our algorithm identifies correctly when no JT organization exists for a given subenv decomposition. Further research is needed for distributed revision of the subenv decomposition under the condition where no JT exists, while preserving agent privacy as much as possible.

Acknowledgement. We thank anonymous reviewers for their helpful comments. Financial support through Discovery Grant from NSERC, Canada is acknowledged. 


\section{References}

1. Brito, I., Meseguer, P.: Cluster tree elimination for distributed constraint optimization with quality guarantees. Fundamenta Informaticae 102(3-4), 263-286 (2010)

2. Gmytrasiewicz, P., Durfee, E.: Rational communication in multi-agent environments. Auto. Agents and Multi-Agent Systems 4(3), 233-272 (2001)

3. Koller, D., Milch, B.: Multi-agent influence diagrams for representing and solving games. In: Proc. 17th Inter. Joint Conf. on Artificial Intelligence, pp. 1027-1034 (2001)

4. Maestre, A., Bessiere, C.: Improving asynchronous backtracking for dealing with complex local problems. In: Proc. 16th European Conf. on Artificial Intelligence, pp. 206-210 (2004)

5. Modi, P., Shen, W., Tambe, M., Yokoo, M.: Adopt: asynchronous distributed constraint optimization with quality guarantees. Artificial Intelligences 161(1-2), 149-180 (2005)

6. Paskin, M., Guestrin, C., McFadden, J.: A robust architecture for distributed inference in sensor networks. In: Proc. Information Processing in Sensor Networks, pp. 55-62 (2005)

7. Petcu, A., Faltings, B.: A scalable method for multiagent constraint optimization. In: Proc. 19th Inter. Joint Conf. on Artificial Intelligence, pp. 266-271 (2005)

8. Valtorta, M., Kim, Y., Vomlel, J.: Soft evidential update for probabilistic multiagent systems. Int. J. Approximate Reasoning 29(1), 71-106 (2002)

9. Vinyals, M., Rodriguez-Aguilar, J., Cerquides, J.: Constructing a unifying theory of dynamic programming DCOP algorithms via the generalized distributive law. J. Autonomous Agents and Multi-Agent Systems 22(3), 439-464 (2010)

10. Xiang, Y.: Probabilistic Reasoning in Multiagent Systems: A Graphical Models Approach. Cambridge University Press, Cambridge (2002)

11. Xiang, Y., Hanshar, F.: Multiagent expedition with graphical models. Inter. J. Uncertainty, Fuzziness and Knowledge-Based Systems 19(6), 939-976 (2011)

12. Xiang, Y., Mohamed, Y., Zhang, W.: Distributed constraint satisfaction with multiply sectioned constraint networks. accepted to appear in International J. Information and Decision Sciences (2013)

13. Xiang, Y., Srinivasan, K.: Construction of privacy preserving hypertree agent organization as distributed maximum spanning tree. In: Zä̈ane, O., Zilles, S. (eds.) AI 2013. LNCS, vol. 7884, Springer, Heidelberg (2013) 